\title{
Recursos de Virtualidade integrados com Realidade Aumentada em Dispositivos Móveis para auxiliar Estudantes na Aprendizagem de Física
}

\author{
Fabrício Herpich $^{1}$, Renan Luigi Martins Guarese ${ }^{2}$, \\ Liane Margarida Rockenbach Tarouco ${ }^{1}$ \\ ${ }^{1}$ Centro Interdisciplinar de Informática na Educação \\ ${ }^{2}$ Instituto de Informática \\ Universidade Federal do Rio Grande do Sul (UFRGS) \\ Porto Alegre - RS - Brasil \\ \{fabricio.herpich, renanghp\}@gmail.com, liane@penta.ufrgs.br
}

\begin{abstract}
Resumo. Este artigo apresenta um protótipo de aplicativo para dispositivos móveis que emprega recursos de realidade aumentada para ensejar a aprendizagem de estudantes em física. Para tanto, proporciona aos seus usuários a interação com recursos educacionais multimídias, tais como objetos $3 D$ e simulações. Outra função própria do aplicativo permite que os usuários combinem a utilização do protótipo com recursos existentes em laboratórios virtuais de física desenvolvidos na plataforma OpenSimulator, no qual os estudantes são representados por avatares e possuem à disposição cenários para navegarem e aprenderem sobre os conteúdos de física, bem como utilizar o aplicativo para interagir com os experimentos físicos que são apresentados.
\end{abstract}

\section{Cenário de uso}

Atualmente uma série de estudos tem investigado formas de aprimorar o aprendizado no nível superior. No entanto, na opinião dos autores deste trabalho a educação brasileira tem apresentado lacunas no aprendizado de base, o qual é definido pela Base Nacional Comum Curricular e estabelece um conjunto de aprendizagens essenciais que todos os alunos devem desenvolver ao longo das etapas e modalidades da educação básica. Este ponto de vista fundamenta-se nos dados do Índice de Desenvolvimento da Educação Básica (IDEB), no qual pode-se verificar que os resultados dos anos finais do ensino fundamental, no período dos anos de 2013 e 2015, em nenhuma das quatro dependências administrativas (Estadual, Municipal, Pública e Privada) foram alcançadas as metas estabelecidas para os respectivos anos.

Nesta perspectiva, envolver os estudantes no processo de aprendizagem em disciplinas da Educação Básica torna-se algo fundamental e tem se configurado como um desafio aos educadores das escolas brasileiras. Isso se motiva uma vez que, nos dias de hoje, a atenção dos aprendizes já não é mais atraída com os mesmos recursos que eram utilizados no passado, sendo necessário inovar na educação básica e fazer com que os alunos se sintam engajados no processo de aprendizagem. Uma alternativa que tem se apresentado viável para tornar os estudantes mais participativos consiste na introdução de tecnologias na sala de aula, aproximando os conteúdos e atividades acadêmicas aos meios nos quais os alunos estão 
inseridos nos dias de hoje, e.g., o uso de dispositivos móveis e redes sociais para envolvê-los em ambientes virtuais de aprendizagem, jogos educacionais, entre outros recursos educacionais.

O uso dos recursos de realidade virtual e aumentada em dispositivos móveis está entre as abordagens viáveis para envolver os estudantes no processo da educação básica, uma vez que possibilita explorar os seus recursos virtuais integrados com os elementos do mundo real com um viés educacional. Tais elementos podem ser acrescidos da apresentação em escala de modelos tridimensionais e outras funcionalidades, bem como a utilização dos recursos próprios dos aparelhos portáteis, tais como câmera, conectividade e localização.

Já referente ao contexto empregado neste estudo, o aproveitamento da realidade aumentada proporciona aos estudantes a visualização de diversos fenômenos que ocorrem na natureza, tais como as leis da fisica e reações químicas. Essas manifestações muitas vezes são abstratas e, assim, não poderiam ser vistas pelos alunos, ou então microscópicas, sendo apenas visualizáveis com o uso de equipamentos de ampliação. Este tipo de situação tende a comprometer o pleno entendimento dos alunos sobre o assunto que está sendo demonstrado, em virtude de não conseguirem refletir e idealizar o conteúdo de maneira adequada e serem obrigados a abstrair de forma parcial ou limitada diversos conceitos físicos que se manisfestam na natureza.

$\mathrm{O}$ aplicativo de realidade aumentada apresentado neste artigo consiste em um módulo para dispositivos móveis do Ambiente Virtual de Aprendizagem e Trabalho Acadêmico Remoto (AVATAR) (2017), projetado na UFRGS e financiado pela CAPES, o qual tem como objetivo implementar formas de oportunizar a aprendizagem por meio de laboratórios virtuais através de mundos virtuais, construídos na plataforma Open Simulator (OpenSim). Para isso, congrega o uso de diversas tecnologias que viabilizam a interação dos seus usuários com simulações interativas, conteúdos didáticos, entre outros recursos pedagógicos.

\section{Ambiente Virtual AVATAR}

O AVATAR apresenta laboratórios virtuais capazes de auxiliar os estudantes no processo de aprendizagem de conteúdos relacionados às leis da fisica e aos fenômenos e processos que ocorrem na natureza e que possuem relações com os princípios físicos conhecidos pela humanidade. Para a construção destes laboratórios virtuais, adotou-se o uso de mundos virtuais como o OpenSim, o qual possibilita o desenvolvimento de cenários em três dimensões, simulações interativas e a inserção de diversos objetos pedagógicos que permitem demonstrar aos estudantes a ocorrência dos fenômenos e os conceitos físicos aos quais estão interligados.

Nos laboratórios virtuais o estudante está representado por meio de um avatar, aspecto que o permite interpretar um personagem e sentir-se imerso no ambiente virtual durante a sua interação com os objetos de aprendizagem ali dispostos. Os cenários construídos e apresentados aos estudantes foram preparados observando uma teoria de aprendizagem condizente com o conteúdo que buscou-se apresentar aos estudantes nos laboratórios virtuais, sendo assim abordou-se o uso da teoria de aprendizagem experiencial, fundamentando-se no Ciclo de Kolb (1984), a qual enseja que os estudantes percorram quatro etapas durante o processo de aprendizagem: a) experiência concreta: os aprendizes executam mais de uma vez as simulações existentes nos laboratórios virtuais; b) observação e reflexão: os alunos analisam e repensam sobre o que foi visto na simulação, com a intenção de 
VI Congresso Brasileiro de Informática na Educação (CBIE 2017)

Anais dos Workshops do VI Congresso Brasileiro de Informática na Educação (WCBIE 2017)

constatar o que foi observado; c) conceituação abstrata: os estudantes buscam entender o que foi visualizado e assimilar o porquê da simulação executar determinadas ações; e, por fim, d) experimentação ativa: os alunos procuram replicar os conceitos que identificaram nos laboratórios virtuais em outras situações do seu dia-a-dia, experienciando e observando se as mesmas leis da fisica se aplicam em diferentes situações. Conforme pode ser constatado nas quatro etapas descritas acima, a aprendizagem experiencial além de ser uma excelente abordagem para a instrução e ensino de estudantes, também faz com que os aprendizes sejam mais ativos no transcorrer de todo este processo, uma vez que são os próprios alunos que executam os procedimentos, a partir de seu interesse e curiosidade em descobrir os princípios que estão relacionados às ocorrências que the foram apresentadas nos laboratórios virtuais.

Em se tratando dos recursos educacionais aos quais os estudantes interagiram durante a navegação nos laboratórios virtuais, foram desenvolvidos diversos experimentos com o intuito de demonstrar a ocorrência dos fenômenos físicos em situações do dia-a-dia, bem como em situações encontradas em laboratórios reais de física. Para envolver os estudantes com a aprendizagem inerente aos experimentos, um estudo realizado por Herpich et al. (2016) identificou a necessidade da construção de cenários atrativos com objetos pedagógicos altamente interativos, que possibilitem ao usuário não só interagir, mas também alterar as variáveis de execução dos experimentos, com conteúdos educacionais simples e objetivos, que ofereçam feedback constante e permitam observar de forma clara os ganhos que as simulações em questão oferecem à sua instrução formativa.

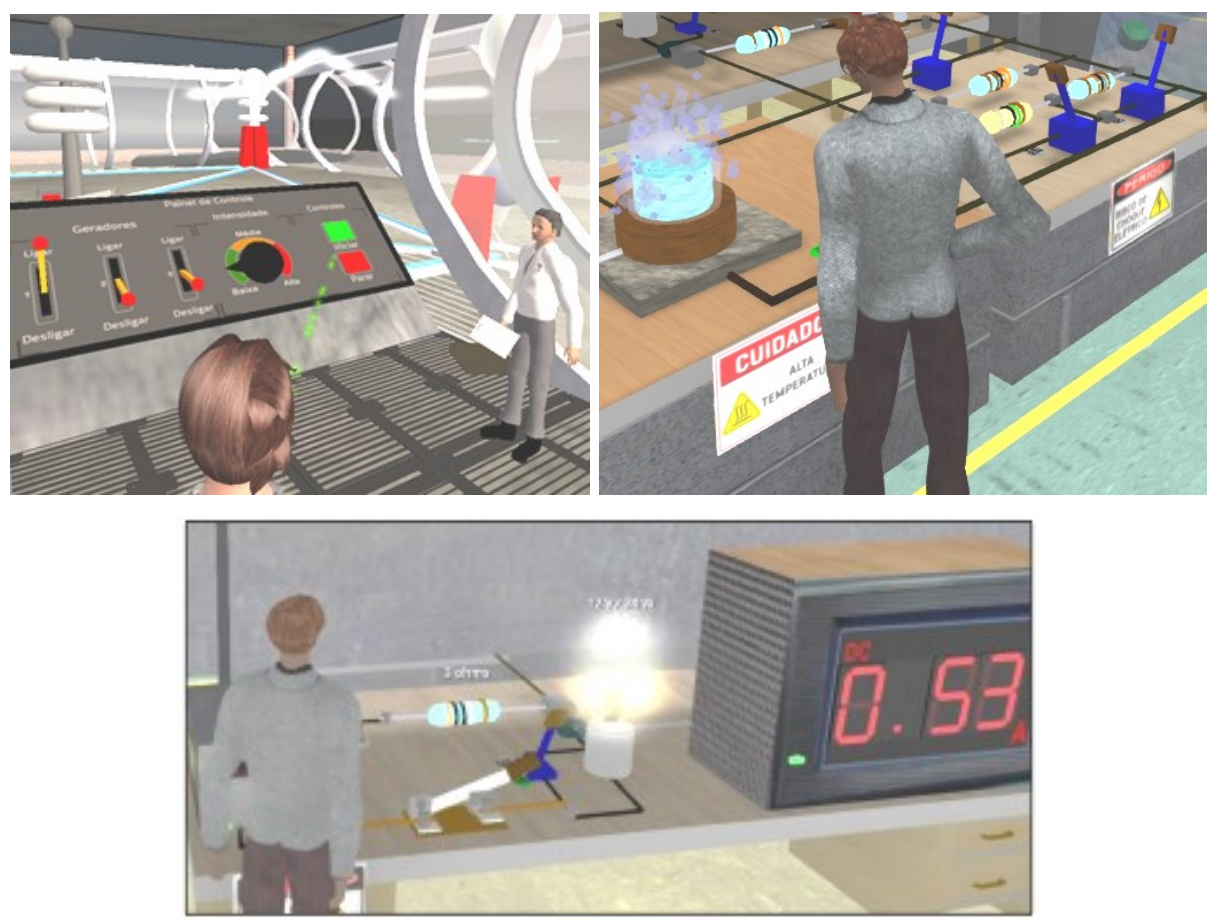

Figura 1. Simulações de Física nos Laboratórios Virtuais

Neste sentido, é possível observar três simulações existentes nos laboratórios (Figura 1), em que os estudantes conseguem observar a formação de arcos voltaicos por meio de uma bobina de Tesla, a ebulição da água através do efeito de Joule, bem como realizar e interagir na configuração de um circuito elétrico. Estes exemplos foram implementados através 
VI Congresso Brasileiro de Informática na Educação (CBIE 2017)

Anais dos Workshops do VI Congresso Brasileiro de Informática na Educação (WCBIE 2017)

da codificação de scripts na linguagem nativa do OpenSim, denominada de OpenSimulator Scripting Language (OSSL), que foram associados aos objetos 3D construídos nos softwares Blender e SketchUp, aspectos que viabilizaram demonstrar de uma maneira fidedigna os princípios físicos aos estudantes. Outro recurso disponibilizado aos estudantes consiste num agente conversacional que está representado no laboratório virtual na mesma forma dos estudantes, através de um avatar, e denominado de Agente Tutora para o Ensino e Navegação no Ambiente virtual imersivo (ATENA), que apresenta a capacidade de responder os questionamentos dos alunos sobre os conteúdos de física.

A fim de alcançar um maior número de usuários e tornar-se amplamente disponível em diferentes plataformas, o AVATAR foi desenvolvido em dois módulos distintos, nos quais o primeiro concentra-se no uso de computadores para o acesso dos estudantes aos laboratórios virtuais e o segundo aborda o uso de dispositivos móveis. Neste artigo o enfoque será destinado ao módulo para dispositivos portáteis, que será descrito na próxima seção.

\section{Desenvolvimento do Módulo para Dispositivos Móveis}

Ao longo das últimas seções foram apresentados e discutidos os assuntos que englobam o AVATAR. No entanto, conforme mencionado anteriormente, o enfoque deste artigo consiste em apresentar o aplicativo para dispositivos móveis e, para isso, preparou-se um diagrama da arquitetura que demonstra as interações mais relevantes que os estudantes podem realizar durante a utilização do aplicativo para dispositivos móveis (Figura 2).

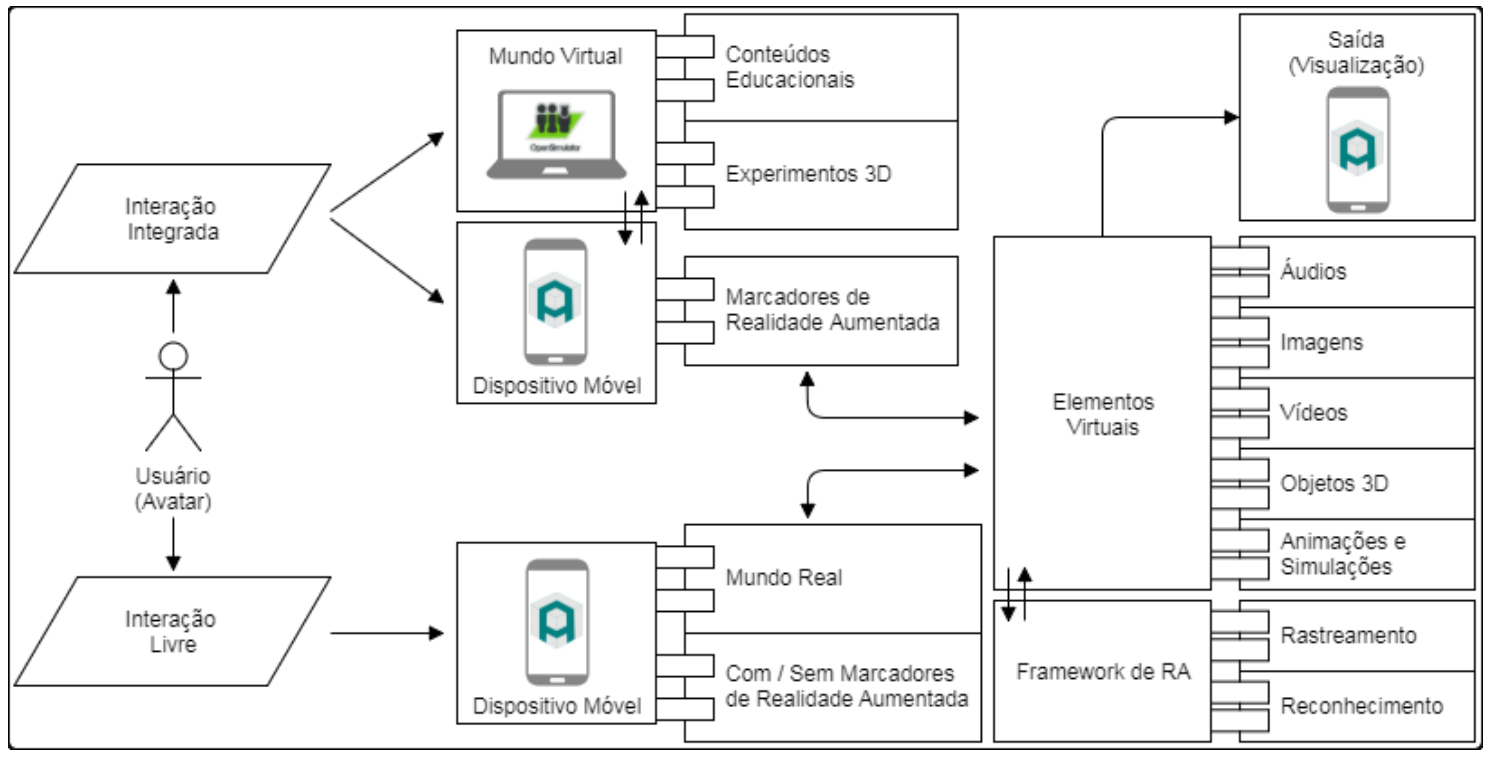

Figura 2. Arquitetura do Aplicativo de Realidade Aumentada

Como pode ser observado na Figura 2, o estudante tem a sua disposição duas formas de interação com o aplicativo de realidade aumentada, as quais são "Integrada" e "Livre":

A primeira opção consiste no estudante realizar o acesso aos laboratórios virtuais do AVATAR e utilizar os marcadores (no formato de texturas) existentes nestes cenários com a intenção de combinar a visualização dos elementos virtuais de realidade aumentada com o seu dispositivo móvel e os recursos pedagógicos existentes nos laboratórios virtuais, apenas apontando a câmera do dispositivo portátil para a tela do computador e verificando os 
VI Congresso Brasileiro de Informática na Educação (CBIE 2017)

Anais dos Workshops do VI Congresso Brasileiro de Informática na Educação (WCBIE 2017)

objetos educacionais que the são apresentados (Figura 3). Esta opção consiste na maneira tradicional de utilizar os recursos de realidade aumentada, por meio do uso de marcadores (markers), os quais servem como ferramenta de forma similar a QR-Codes, i.e., ao serem reconhecidos por um módulo de visão computacional, retornam informações associadas àquele marcador, que nesta circunstância compreendem aos conteúdos de física.

Já a segunda opção configura-se na interação do aluno com o aplicativo de maneira independente (Figura 7), em que não é necessário o acesso aos laboratórios, permitindo o aprendiz acessar aos objetos educacionais em qualquer lugar e a qualquer momento. Isso habilita o acesso do estudante de maneira fácil, com ou sem o uso de marcadores de realidade aumentada, aspecto que amplia as opções do aluno durante a interação com os recursos pedagógicos. Essa última forma de navegação é possibilitada por meio do uso da tecnologia de Realidade Aumentada markerless (sem marcadores), que consiste na capacidade do aplicativo de visualizar e reconhecer o ambiente real em que o estudante encontra-se por meio da câmera do dispositivo móvel, a fim de apresentar os elementos virtuais integrados a esse ambiente. Além disso, no modo de interação livre o aplicativo também permite que o aprendiz não só utilize marcadores virtuais, mas também impressos, que eventualmente tenha recebido em sala de aula pelo professor, possibilitando que o estudante visualize os elementos virtuais sem a necessidade de realizar acesso aos laboratórios virtuais. Por meio desta funcionalidade, é possível oportunizar ao professor um controle melhor e mais simples sobre os conteúdos disponíveis aos seus alunos em um dado momento.

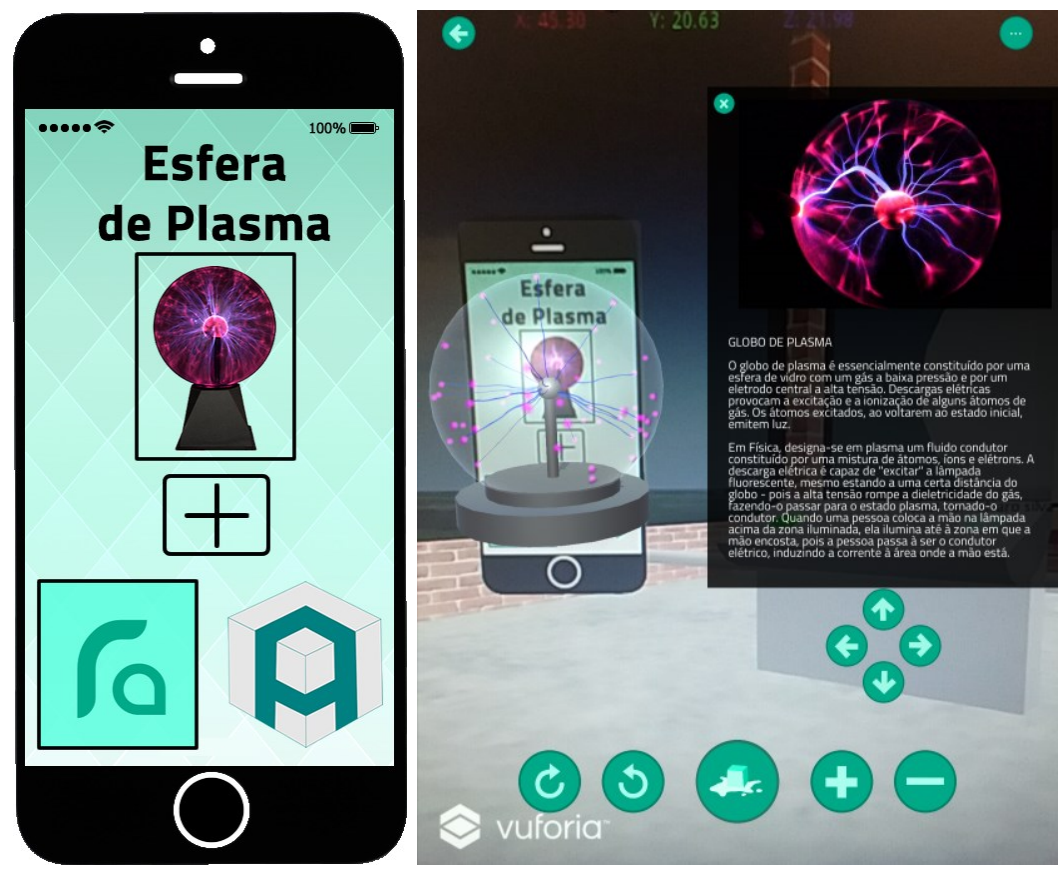

Figura 3. Marcador de Realidade Aumentada e Recurso Virtual

Em relação a implementação do aplicativo de realidade aumentada para dispositivos móveis, foi necessário a integração de diferentes frameworks capazes de oportunizar a construção desta solução educacional. Para tanto, foi utilizada a ferramenta Unity 3D para a implementação do aplicativo e exportação para a plataforma Android, aliada ao uso da linguagem de programação C\#, que permitiu elaborar os comportamentos, através de scripts, 
VI Congresso Brasileiro de Informática na Educação (CBIE 2017)

Anais dos Workshops do VI Congresso Brasileiro de Informática na Educação (WCBIE 2017)

que o aplicativo executa em determinadas situações. Já em relação ao módulo que opera as funcionalidades de realidade aumentada, foi utilizado o framework Vuforia, o qual disponibiliza uma plataforma de visão computacional que permite a construção de experiências interativas e que apresenta diversos recursos relacionados à realidade aumentada (Herpich et al., 2017).

Esta seção buscou apresentar o módulo do AVATAR delineado para a plataforma de dispositivos móveis, demonstrando a utilidade dos recursos de realidade aumentada combinados com laboratórios virtuais, visando oportunizar a aprendizagem dos estudantes em conteúdos relacionados à física e aos fenômenos que ocorrem na natureza por meio de experimentos, objetos $3 \mathrm{D}$, simulações, entre outros recursos educacionais.

\section{Aplicativo de Realidade Aumentada para Aprendizagem de Física}

Para o acesso do estudante ao aplicativo são utilizadas as mesmas credenciais de usuário e senha dos laboratórios virtuais, que uma vez preenchido de maneira correta, habilita ao aprendiz interagir com as funcionalidades disponíveis no aplicativo de realidade aumentada.

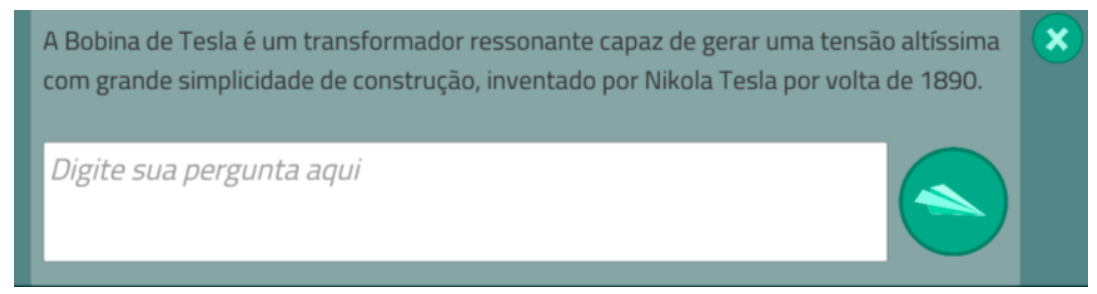

Figura 4. Chatterbot disponível aos Estudantes

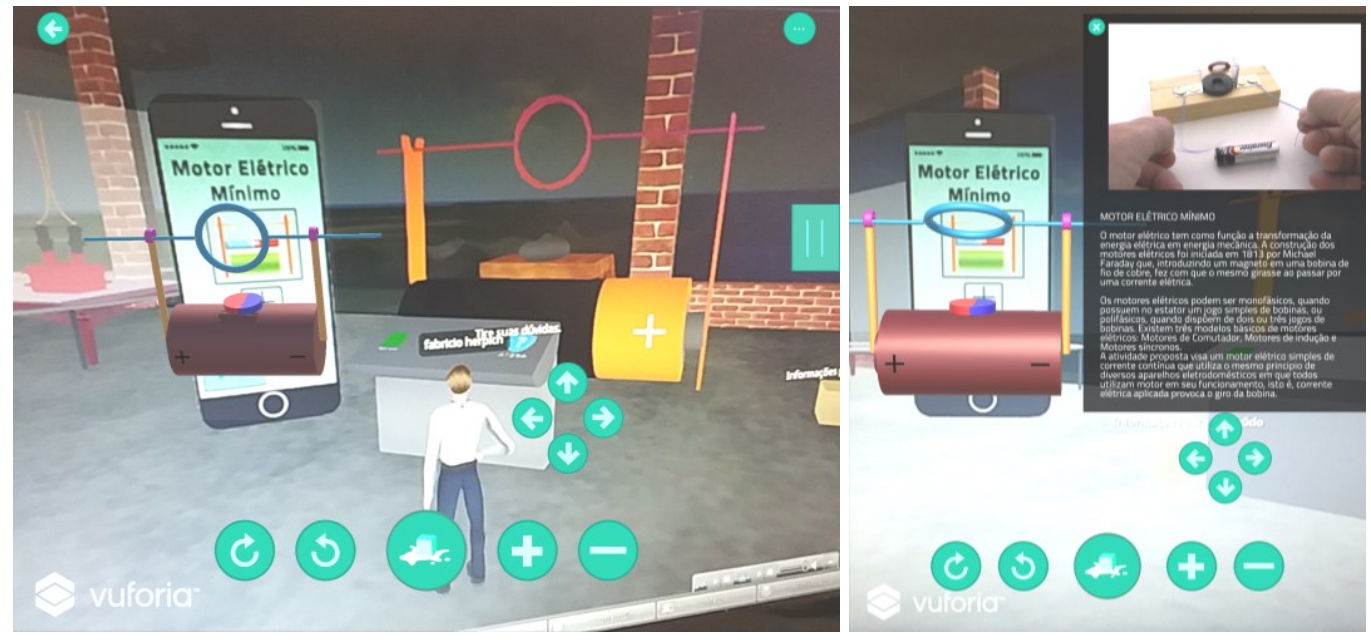

Figura 5. Estudante utilizando o Aplicativo no Modo Integrado

Neste sentido, é possível elencar quatro recursos elementares que o aplicativo contempla: 1) na Figura 4 é apresentada a opção relacionada ao chat, que eventualmente o estudante pode utilizar para realizar uma conversação com o chatterbot (robô conversacional) ATENA e solucionar suas dúvidas sobre os experimentos de física; 2) na Figura 5 é exposta a opção em que o aluno utiliza o recurso do modo de navegação integrado, em que utiliza o seu dispositivo portátil junto aos laboratórios virtuais, para então visualizar os recursos educacionais; 3) a Figura 6 demonstra o inventário particular do 
VI Congresso Brasileiro de Informática na Educação (CBIE 2017)

Anais dos Workshops do VI Congresso Brasileiro de Informática na Educação (WCBIE 2017)

estudante, que contém todos os recursos educacionais que o aprendiz selecionou como favorito, com a intenção de visualizá-los posteriormente e mantê-los em seus registros; e, por fim, 4) a Figura 7 é relacionada à interação no modo livre, no qual o estudante consegue interagir com os experimentos sem o uso de marcadores e a necessidade de acesso aos laboratórios virtuais, integrando os elementos virtuais ao cenário do mundo real.

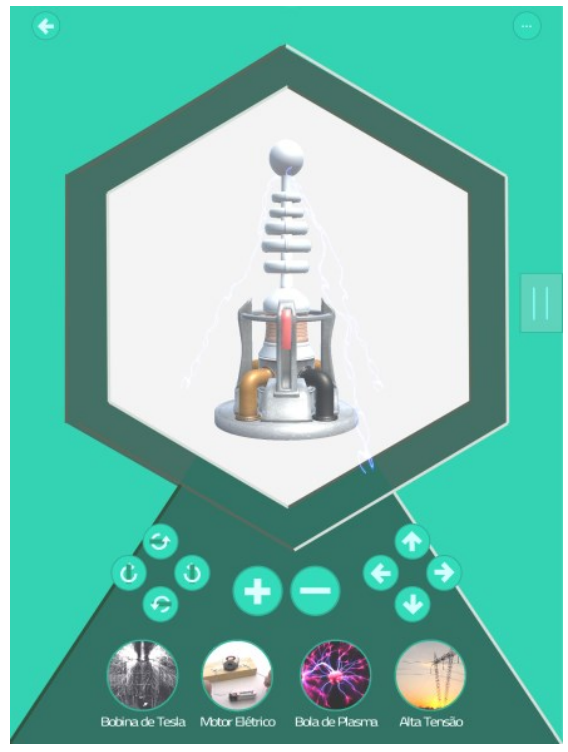

Figura 6. Inventário do Estudante

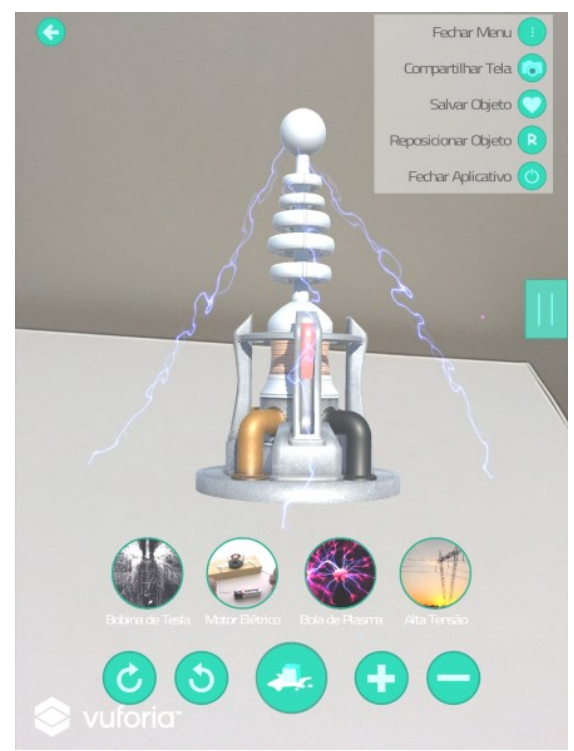

Figura 7. Interação no Modo Livre

O aplicativo também possibilita ao estudante visualizar notas explicativas sobre o experimento apresentado, elucidando os princípios físicos presentes no recurso pedagógico em questão (Figura 3). Outras opções disponíveis são apresentadas na Figura 7, consistem na possibilidade de compartilhar em redes sociais o que está sendo exposto, salvar o objeto como favorito e reposicionar o objeto. $\mathrm{O}$ estudante também tem à sua disposição as opções de aumentar e diminuir os recursos pedagógicos, movê-los e girá-los na tela do dispositivo móvel para visualizá-los de outros ângulos. Há, ainda, o botão "congelar objeto", que permite ao usuário fixá-lo na posição atual em relação à tela do dispositivo, possibilitando a ele que o reposicione no espaço e, ao "descongelar" o objeto, mantê-lo nessa nova posição em relação ao mundo real, desafixando-o de sua relação com a tela. Essa opção dispõe ao usuário uma nova forma de realizar transformações espaciais, incluindo rotações, escalas e translações.

\section{Considerações finais}

Este trabalho apresentou um aplicativo de realidade aumentada capaz de possibilitar aos seus usuários o acesso a diversos recursos educacionais, que podem ser visualizados tanto em seus dispositivos móveis como em computadores, por meio dos laboratórios virtuais construídos na plataforma de mundos virtuais OpenSim.

Os autores deste estudo têm investigado a hipótese de que combinando ambas as tecnologias, realidade virtual e aumentada, é possível implementar recursos de grande potencial para a educação, em que os estudantes obterão um ganho em sua aprendizagem, uma vez que conseguirão visualizar os experimentos de fisica e materializar os princípios físicos envolvidos, os quais muitas vezes não são suficientemente claros nos livros para a 
abstração dos aprendizes, bem como poderão acessar esses recursos através de seus dispositivos móveis em qualquer hora e lugar.

Além disso, em se tratando da interdependência entre os laboratórios virtuais e o aplicativo para dispositivos móveis, foram conduzidos testes preliminares que permitem afirmar que o uso apenas do aplicativo possibilita envolver os estudantes com os conteúdos vistos em sala de aula. Essa afirmação é baseada em um estudo piloto realizado com 77 alunos do sexto ano do Colégio Militar de Porto Alegre, no qual o aplicativo em questão foi adaptado para a área de geografia e utilizado para realizar uma atividade de orientação. $\mathrm{Na}$ oportunidade, os estudantes colocaram em prática os conhecimentos de orientação e localização, através de uma atividade gamificada, que oportunizou encontrarem pistas espalhadas pela instituição e utilizarem o aplicativo com os recursos de realidade aumentada para desvendarem as novas coordenadas da atividade e conquistarem as recompensas conforme o avançar do exercício.

\subsection{Trabal hos Futuros}

Com a intenção de aprimorar o protótipo de aplicativo e torná-lo apto a ser integrado ao cotidiano dos estudantes, no que tange aos conteúdos de física, como etapas futuras será considerado o desenvolvimento de novos recursos educacionais (e.g. simulações e experimentos tridimensionais), buscando combiná-los com as características inovadoras da realidade aumentada (e.g. apresentação de recursos virtuais sem o uso de marcadores e alvos de realidade aumentada geolocalizados).

Também está sendo desenvolvida a ampliação da base de conhecimentos do agente conversacional, a fim de tornar a ATENA capaz de responder os alunos sobre tópicos de física. Assim como está sendo implementada uma plataforma web que irá habilitar aos professores à importação de forma fácil dos seus recursos educacionais (e.g. objetos 3D, vídeos, apresentações, entre outros), de modo integrado ao aplicativo de realidade aumentada.

\section{Referências}

AVATAR (Página Oficial). Acessado em Junho de 2017, disponível em: http $/ /$ www.ufrgs.br/avatar.

Herpich, F., Guarese, R. L. M., Tarouco, L. M. R. (2017). A comparative analysis of Augmented Reality Frameworks aimed at the development of Educational Applications. Creative Education, Vol.8 No.9, p. 1433-1451. https//doi.org/10.4236/ce.2017.89101

Herpich, F., Nunes, F. B., Cazella, S. C., \& Tarouco, L. M. R. (2016). Mineração de Dados Educacionais: uma análise sobre o engajamento de usuários em mundos virtuais. In $\mathrm{V}$ Congresso Brasileiro de Informática na Educação (CBIE), p. 910-919.

Instituto Nacional de Estudos e Pesquisas Educacionais Anísio Teixeira (INEP). Índice de Desenvolvimento da Educação Básica (IDEB). Acessado em Junho de 2017, disponível em: http://sistemasideb.inep.gov.br/resultado/.

Kolb, D. (1984). Experiential Learning: Experience as the Source of Learning and Development. Prentice Hall, Englewood Cliffs. 